\title{
Ugarit: Translation Alignment Visualization
}

\author{
Tariq Yousef \\ NLP Group, Institute for Computer Science, University Of Leipzig, Germany \\ E-mail: tariq@informatik.uni-leipzig.de
}

\begin{abstract}
Ugarit is a web-based tool for manual translation alignment of parallel texts. The aim was to build a user-friendly interactive interface to visualize aligned texts and collect training data in the form of translation pairs to be used later for training an automatic translation alignment system at the word/phrase level or as a gold standard to evaluate machine translation systems. The tool is now widely used for learning new languages, especially historical languages, and as a reading environment for parallel texts. In the next sections, we present the related works and similar projects; then, we give a short overview of the visualization techniques used to present the alignment results. Further, we explain how we could derive the translation graph from the aligned translation pairs. Finally, we discuss the usage limitations of Ugarit and the improvement suggestions in addition to our future development plans.
\end{abstract}

Index Terms-Translation Alignment, Data Visualization, Manual Alignment, Translations Graph.

\section{INTRODUCTION}

Translation alignment is one of the main tasks in Digital Humanities and Natural Language Processing. It is defined as comparing two texts in different languages to find translation correspondences among the textual units in the source, and target texts [1]. Translation alignment can be performed at various granularity levels depending on the project's context or the research purpose.

Several automatic approaches have been developed to perform the alignment at different levels [1][2][3][4]. However, most suggested models employ unsupervised statistical methods to generate alignment probabilities distribution between the source and target textual units. In general, The accuracy of the automatic alignment varies according to multiple factors such as text type and length, size of the corpus, and translation quality and consistency. In contrast, manual alignments are accurate and reliable since they are produced by scholars and experts. Manual alignment is expensive in terms of time and resources; users working on it should know both languages.Ugarit is a crowd-sourcing project that enables users to create translation alignments at the word or phrase level; The resulting translation pairs can be re-used in future machine translations or to create dynamic lexica and translation memories.

Ugarit was initially designed to visualize the automatically aligned texts available at Perseus Digital Library [5] and

LEVIA'19: Leipzig Symposium on Visualization in Applications 2019 collect training data in form of translation pairs to implement a statistical translation alignment system for historical languages, mainly Ancient Greek, Latin, and Persian, for which few to none aligned data sets exist. Ideally, historical languages are closed systems with a finite number of words and minimal change in the foreseeable future. Therefore, it should be possible to create adequately efficient automated statistical machine alignment methods based on a relatively small training data set.

Since the tool was made public, the number and variety of languages included by the users has steadily increased and has gone far beyond the original intent: at the moment this paper is being written, 36 languages are included in Ugarit, and there are 295 active users, and about 23,500 parallel texts. The development of Ugarit started in 2017 at the AvH Chair for digital humanities at the University of Leipzig, under Prof. Gregory Crane's supervision and in collaboration with Chiara Palladino and Maryam Foradi.

In the next sections, we review similar and related works, then we explain how the manual alignment can be done in Ugarit. Next, we show Ugarit's various visualization techniques to visualize the translation alignments and the dynamic lexicon search results; then, we present the translation graph. Finally, we discuss the possible improvements and new features we intend to add to the next release of Ugarit.

\section{RELATED WORKS}

The related works can be grouped into three main groups; the first one contains tools and projects that offer an annotation interface to collect translation pairs to be used in further development without any interest in visualizing the alignment. The second group provides both the annotation and the visualization interface. In comparison, tools in the third group can be considered analytical tools that offer various visualization approaches to present the alignment at different levels.

From the first group, we can mention the Blinker Project [6], which has developed the first annotation tool for manual text alignment to align different versions of the Bible in French and English at the word level. The Linguistic Data Consortium (LDC) has also developed the LDC Word Aligner to perform the manual word alignment for Arabic-English and ChineseEnglish parallel texts from newswire and broadcast mainly [7]. Furthermore, TagAlign [8] allows users to annotate bilingual texts with a pre-defined tagset and create manual alignment at 
the sentence level. The tool was initially developed to align Brazilian, Portuguese, and English parallel Texts.

Alpheios [9], which falls in the second group, can be considered the first public web-based manual alignment tool for translation alignment. Alpheios enables users to create their alignment manually at the word level and offers two approaches for visualizing the aligned texts, the side-by-side view and the interlinear text view [10].

Visualization of aligned texts was the subject of interest and research in recent years; many tools have been developed for this purpose. Various approaches have been utilized to visualize the text alignment at different levels. JuXtacommons[11] and Versioning Machine use the side-by-side approach to visualize the alignment between texts in the same language at word level. TRAViz[12] and Stemmaweb[13] use the variant graph to represent the alignment between text variants. Alignment Map [14] uses the parallel view to visualize translation alignment. It shows the base text and its translation connected by lines, the color and the thickness of the line reflects the length of the aligned segments in words. Iteal[15] introduced the Meso reading to visualize the alignment between parallel texts at line and word level. Other tools like CATView[16] and iAligner[17] use the tabular view to show the positions of similarity and differences between parallel texts.

\section{Alignment Workflow}

Annotation is the process of adding information to a text at some level [18], and we call it Manual Annotation when humans perform it. Manual Annotation is a nontrivial task, and it requires a lot of focus and attention. Like any chore, it becomes boring after a short while. Manual translation alignment, in particular, is a daunting task; it requires moving eyes between two texts in different languages and finding translation correspondences and linking them.

When we designed Ugarit Interface, we tried to make it easy to use even for users with no experience with annotation tools. The first step is creating an account on Ugarit or logging in if the user has already created an account and verified it. To start a new alignment, users have to choose between two or three text alignments. Besides, users can then upload their corpus in plain text format or use the canonical text service (CTS) URNs to import texts from the Perseus Digital Library CTS ${ }^{1}$ repositories [19]. Next, the languages must be selected. Texts will be tokenized and prepared for alignment; Ugarit uses whitespace and punctuation tokenizer to split the text into a sequence of tokens.

The alignment process is designed to be as simple as possible; we tried to minimize the mouse clicks needed to create and save the aligned tokens. In the case of bilingual text alignment, the panel is splited into three main columns. Two columns for the parallel texts and the third for displaying the aligned translation pairs. A progress bar is located on the top of each language panel to show the alignment coverage so far. To align a translation pair, users have to select the word/phrase from the

\footnotetext{
${ }^{1}$ cts.perseids.org
}

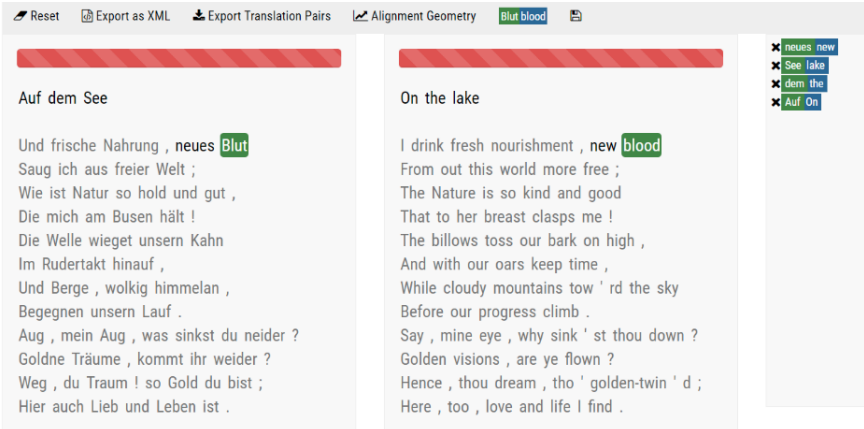

Fig. 1: Ugarit manual texts alignment editor.

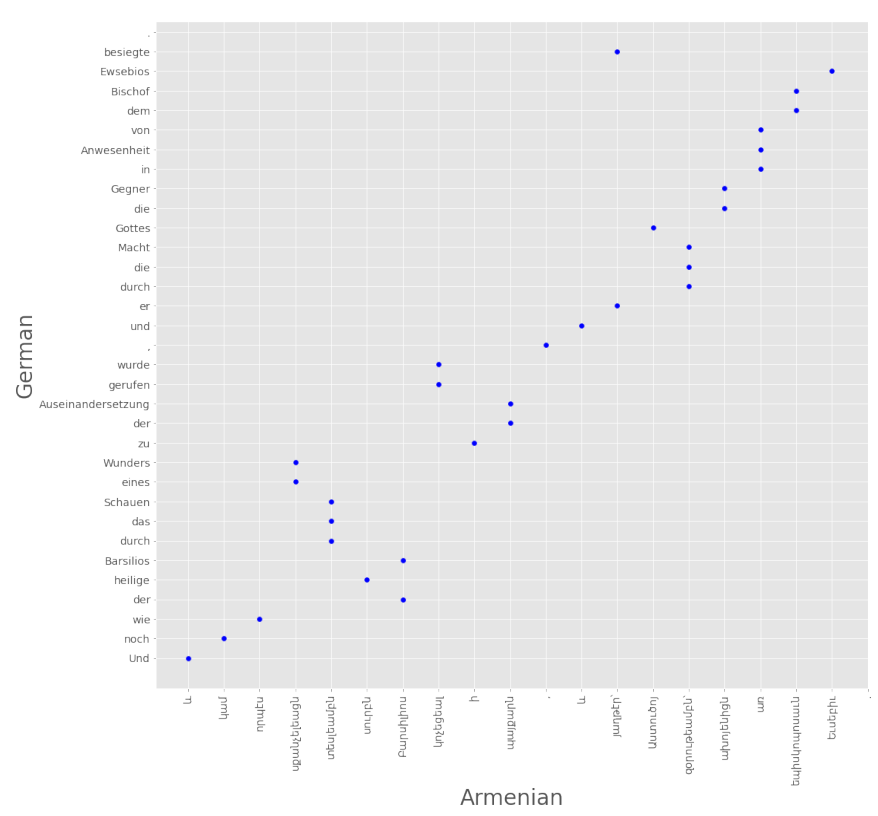

Fig. 2: Alignment of Armenian text with its German translation created by Uta Koschmieder.

original language and then select the corresponding tokens in the translated text (Fig 1). To select a token, the user can click the token, and then it will be highlighted with green. Clicking a selected token will deselect it and remove the highlighting. The paired tokens will be automatically saved when the user starts to align a new pair or clicks on the save icon. On the right side of the editor, users can see all aligned pairs and have the option to edit or delete any pair. Ugarit editor allows users to create all types of alignment one-to-one, one-to-many, many-to-one, and many-to-many alignments. The resulting translation pairs are automatically stored in the database and then exported in XML or tabular format. Furthermore, users can also decide whether the alignment can be publicly visible on the website or not. Once the user saves the alignment, it will appear on the home page in the "New Alignments" panel. Finally, users have to provide some information about the texts such as title, translator, and a short description. Ugarit also offers trilingual alignment, where users can align three parallel texts in three different languages. There is no general alignment guidelines 


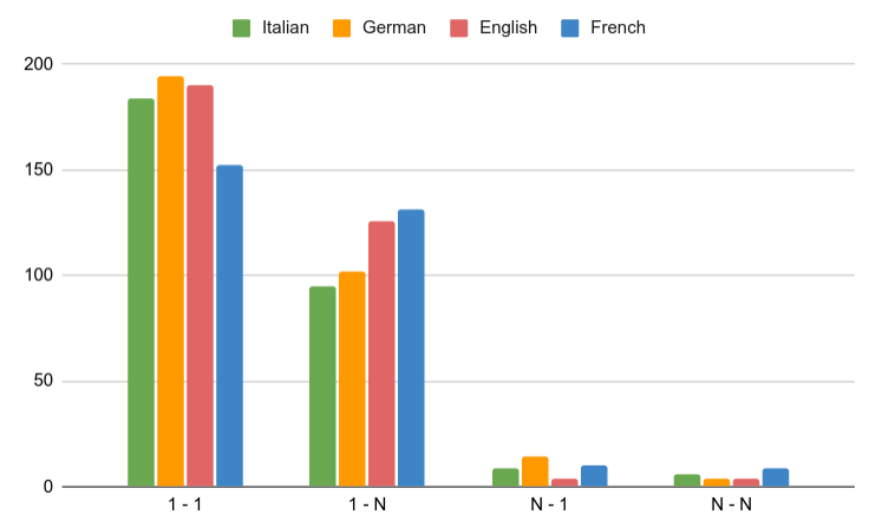

Fig. 3: Alignment types of the translation alignments of an ancient Greek text with its translations in different languages.

provided by Ugarit since it is language- and project-dependent. Every user can follow the guidelines he prefers.

Figure 2 shows the alignment between an Armenian source text ${ }^{2}$ and its German translation as a dot map; the diagonal points indicate one-to-one alignments. The vertical points reflect the one-to-many;

Figure 3 shows the different alignment types of the translation alignments of an ancient Greek text with its translations in Italian, German, English, and French ${ }^{3}$. This distribution of the alignment types differs according to various factors including text languages, text type, text genre, translation type, annotator guidelines.

\section{Visualization TeChniQues}

Visualization of translation alignment plays an important role in understanding and interpreting the relation between texts and their translations. Ugarit offers various approaches to visualize aligned texts and the derived dynamic lexicon.

\section{A. Languages Graph}

The graph is placed on the tool's home page ${ }^{4}$ to give users a quick overview of the languages currently hosted and how they are related to each other, as shown in Fig 4.

Each vertex represents a language. The vertex's size reflects the number of texts in this language in the database. Each language is assigned a different color, and vertices are labeled with the language names in the original form. The connection between two vertices means that there are translation alignments between texts in these two languages, and the thickness of the line reflects the number of aligned translation pairs. Figure 4 we notice the thick blue line between English and Ancient Greek because Ugarit contains a large amount of

\footnotetext{
${ }^{2}$ The alignment is created by Uta Koschmieder and available at: http://ugarit.ialigner.com/text.php?id=877

${ }^{3}$ The alignment of Homer, Iliad, 21.1-53 ancient greek source text and its different translations, alignments are created by Chiara Palladino and avaialable at: http://ugarit.ialigner.com/userProfile.php?userid=3\&tgid=37

${ }^{4} \mathrm{http}: / /$ ugarit.ialigner.com
}

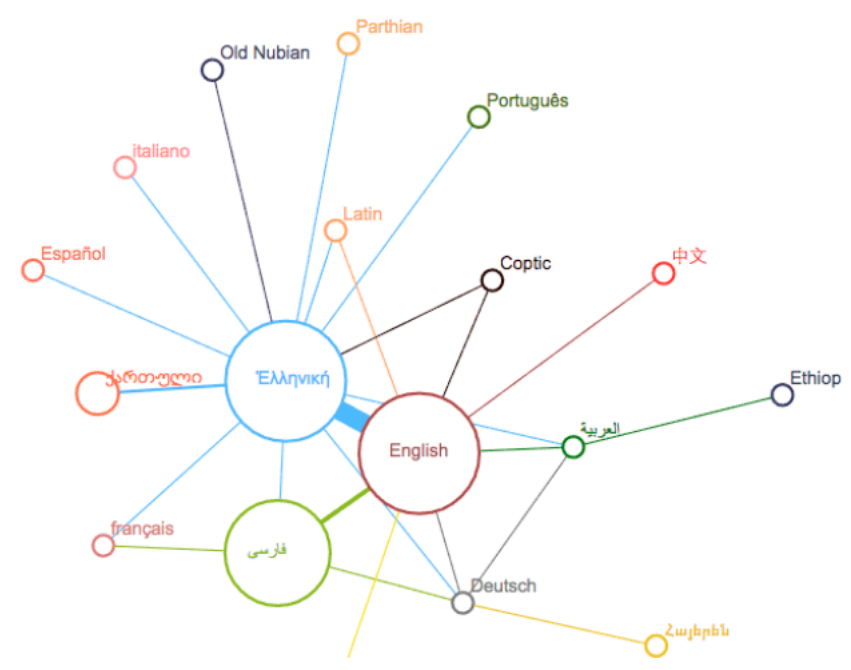

Fig. 4: Languages graph.

English-Ancient Greek automatic aligned texts at the word level provided by Perseus Digital Library [5].

Clicking on a vertex will show all texts in the language it represents. Whereas clicking on a link between two vertices will load all aligned texts in these two languages.

\section{B. Aligned Texts}

The side-by-side view is the most intuitive and straightforward approach to display parallel texts alignment at different levels. It is also widely used to visualize collation and mono-language alignments.

Texts are placed alongside each other, as shown in Fig 5 and Fig 6. A coloring schema is used to distinguish between aligned and unaligned tokens. Since most tokens are aligned, we used black for aligned tokens and red for the unaligned to draw user attention. The alignment between corresponding tokens on the parallel sides is visualized via highlighting. When the user hovers an aligned word, the hovered word/phrase and the paired tokens will be simultaneously highlighted with red color.
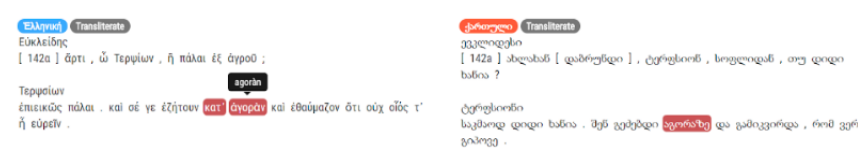

Fig. 5: Side-by-side visualization of a bilingual text.

Ugarit contains texts in various languages with different alphabets. For better readability, especially for new language learners, Ugarit offers an automatic transliteration for nonLatin alphabet languages, which is visible when the pointer hovers the aligned word. This feature is currently available for Greek, Arabic, Persian, Armenian, and Georgian.

A progress bar is located under each text to give the user an overview of how many tokens are aligned and their percentage; the aligned part is colored with green, whereas the unaligned part is colored with red, as we can see in Fig 6. 


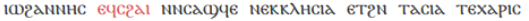

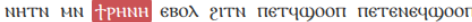

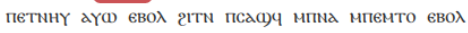
пееропос

\section{EMกVIKi}

'I wávvnৎ eirếnē

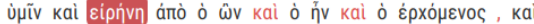

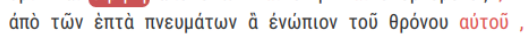

English

John to the seven churches that are in Asia : Grace to you and peace, from him who is and who was and who is to come; and from the seven Spirits that are before his throne ;

Fig. 6: Side-by-side visualization of three parallel texts.

\section{Dynamic Lexicon}

The dynamic lexicon [20] is an automatically derived lexicon from parallel aligned texts at word/phrase level. Triangulation is used to create new dynamic lexica using pivot languages based on the assumption that two expressions are likely to be translations if they are translations of the same word in a third language [21].

Ugarit offers a search function to enable users to look for a word or phrase and get detailed information about how this word/phrase occurs in the text and how it is translated in different languages. Moreover, Ugarit visualizes the dynamic lexicon search results in two approaches:

1) Tree View: is a classic branching view that enables users to navigate the hierarchy to filter the results set until they reach the desired subset. Figure 7 shows how Ugarit visualizes the search results as three levels tree view. The query word is located at the first level as the root word, corresponding translations are grouped by languages, and languages are placed as nodes at the second level. The aligned translations are placed at the third level. The languages nodes are initially collapsed for clarity, and users can expand them to explore the translations by clicking the languages nodes.

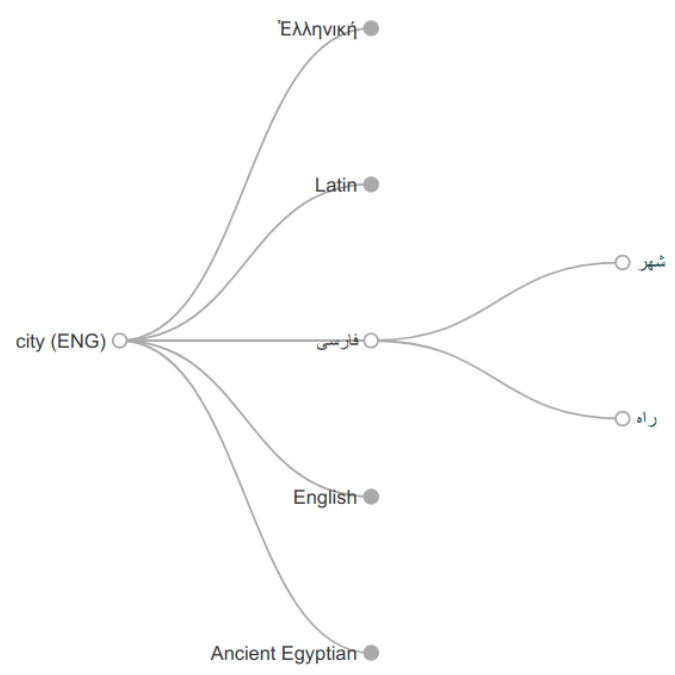

Fig. 7: Tree view visualization of the dynamic lexicon.

2) Radial Cluster Dendrogram: is a visualization approach to show hierarchical data in the form of a rooted tree. Figure 8 shows how Ugarit shows the translation of a given word in the form of a radial dendrogram. The query word is placed as the

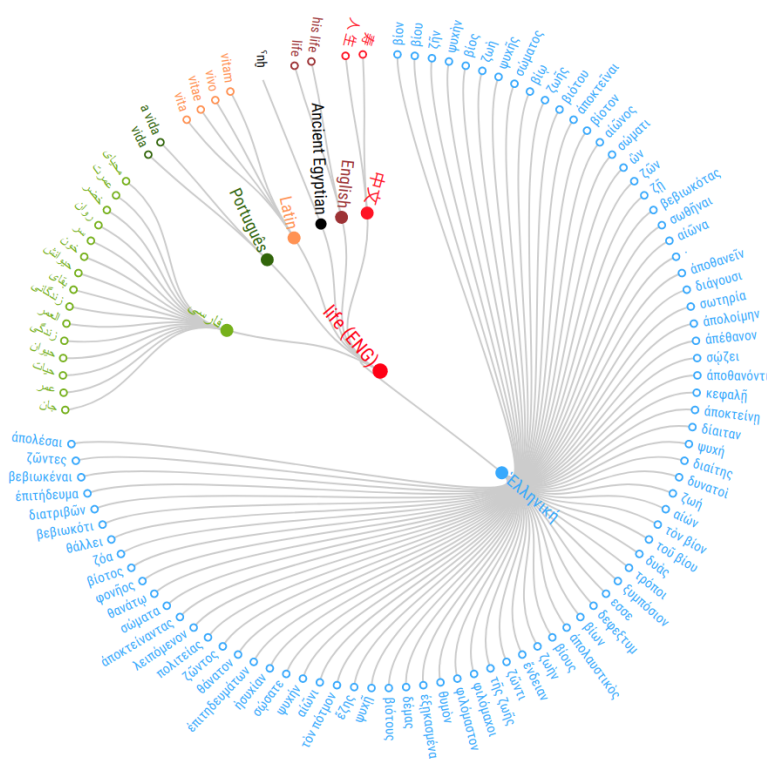

Fig. 8: Radial dendrogram visualization of the dynamic lexicon.

root of the tree in the center of the circle. The children nodes, which are the languages, are located in the inner-most ring. The leaf nodes, which are the translations of the search term clustered by the languages, are placed with equal depth. Each language is colored with a different color.

\section{TRANSLATIONS GRAPH}

Ugarit is characterized by its simplicity and ease of use, and for this reason, it has been used by many users and in several projects, which led to continuous growth in the number of aligned texts and translation pairs. Though the traditional relational database architecture implemented in Ugarit is no longer satisfactory, we realized that we could achieve more benefits if we modeled our data in a more sophisticated way. After experimenting with different models, we came to the conclusion that the graph data structure fits our data best. The proposed model can be described as follows:

Let $G=(V, E)$ is a translations-graph. where $V$ is a set of all words/phrases in the the Ugarit aligned texts, and $E$ is a set of all translation pairs. Let $v_{1} \in V$ is a word/phrase in language $l_{1}$, and $v_{2}$ is word/phrase in language $l_{2} .\left\{v_{1}, v_{2}\right\}$ is a translation pair that occurs $n$ times in the data set. 
Each node $v \in V$ has four attributes, an internal id $i d$, label $t$, language $l$, and $n$ the frequency of this word/phrase in the whole data set $v\{i d, t, l, n\}$.

The translation relation between $v_{1}\left\{i d_{1}, t_{1}, l_{1}, n_{1}\right\}$ and $v_{2}\left\{i d_{2}, t_{2}, l_{2}, n_{2}\right\}$ can be represented as an an edge $e \in E$, $e$ encapsulates a single value $n$ which indicates the frequency of this translation pair in the data set.

We have used Neo4j database [22] to model the translation graph. Since Neo4j does not support indirect relations between nodes, we had to represent each translation pair as two nodes connected with two directed edges, as shown in figures 910 .

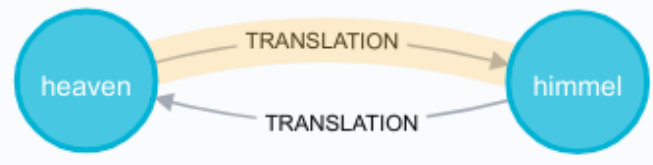

Fig. 9: Graph representation of a translation pair.

\section{A. Translation Clouds}

Visualizing the translation graph as a connected graph was not possible due to the large number of nodes and crossing edges, which would affect the translations' clarity and readability. The solution was to keep the labels and remove the lines between them, resulting in a word cloud representation. Translation cloud shows the translation equivalents to a given word, and these equivalents are extracted from the translation graph up to the fifth level. Figure 11 shows the translations cloud of the root word city. The view consists of three

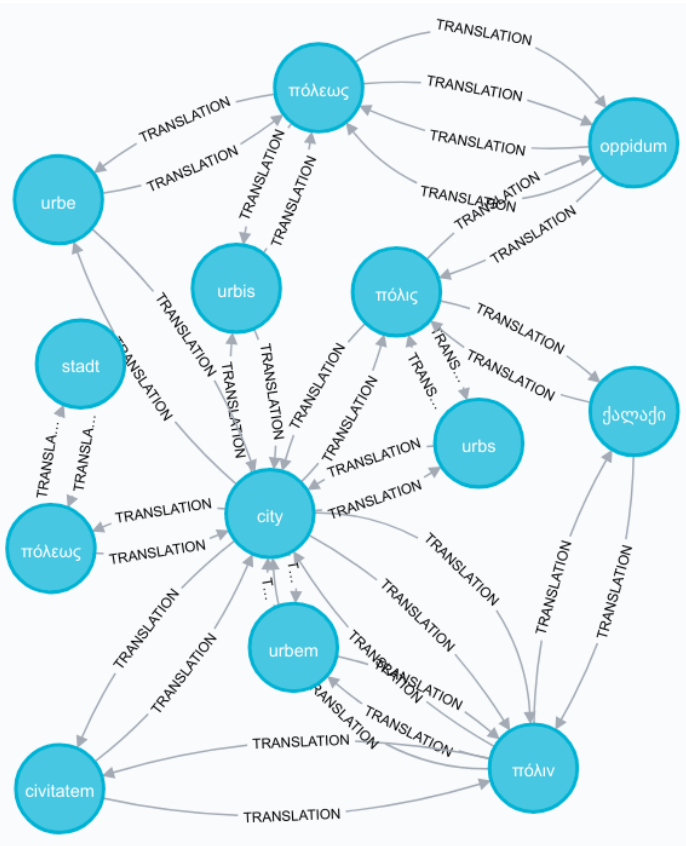

Fig. 10: Translations sub-graph in Neo4j. components, the translation cloud, the languages bar above it, and the concrete translation path on the right side. The search word is located at the cloud center, surrounded by its equivalents in other languages. Further, translation equivalents are grouped by languages, and every language is assigned a unique color. However, languages are listed in the languages bar; clicking on a language label will hide all translation equivalents in other languages and keep the ones in the selected language. Labels are displayed in different font sizes according to the distance to the root word. Since the translations cloud visualizes connected translation equivalents up to the fifth level, the distance to the root varies between 1 to 5 , the lower the distance value, the higher the font size. Moreover, clicking a translation label will show the translation path between the clicked translation and the root word. Moreover, a tooltip is used to show information about the label, such as language and frequency.

Using Neo4j graph database at the back-end to store the translation pairs has shown many advantages; it reduced the response time of search queries, enabled us to perform complex queries, and facilitated the dynamic lexicon production.

\section{FUTURE WORK}

When we created Ugarit, we aimed to visualize the automatically aligned texts and collect training data for automatic alignment systems. Still, Ugarit has also been used in many research projects[23], [24] and for different purposes, especially teaching and learning historical languages [25]. Experts, who have been using Ugarit regularly, have provided us with some ideas and improvement suggestions to enhance the usability of Ugarit. Therefore, new features and functionality should be implemented and developed to keep pace with users' new needs. We can sum them up as follows:

- User roles: the next version of Ugarit will offer different user roles such as expert, instructor, student, which would help create accurate training data by considering the alignments created by experts and instructors, since they are supposed to produce correct and precise alignments. In contrast, students in the learning phase could make some alignment mistakes, and these mistakes should not affect the accuracy of the dynamic lexicon and training data sets.

- Teaching: Further, experts and instructors will be able to create groups, add students to the groups, and create assignments. Instructors can upload these assignments in the form of plain parallel texts; students will be asked to align them with deadlines, with the possibility of uploading the correct alignment to allow the system to evaluate the assignments automatically and give notes to every student.

- Alignments sharing and exporting: in the current version of Ugarit, users can export their results in XML format only. The next version will offer other formats such as JSON and CSV to facilitate the reuse of the alignment in other applications or for other purposes. Further, users will be able to share their alignments and their visualizations via unique URLs. Also, we will provide clear and rich documentation in the form of texts, tutorials, and videos to make the use of Ugarit as simple as 


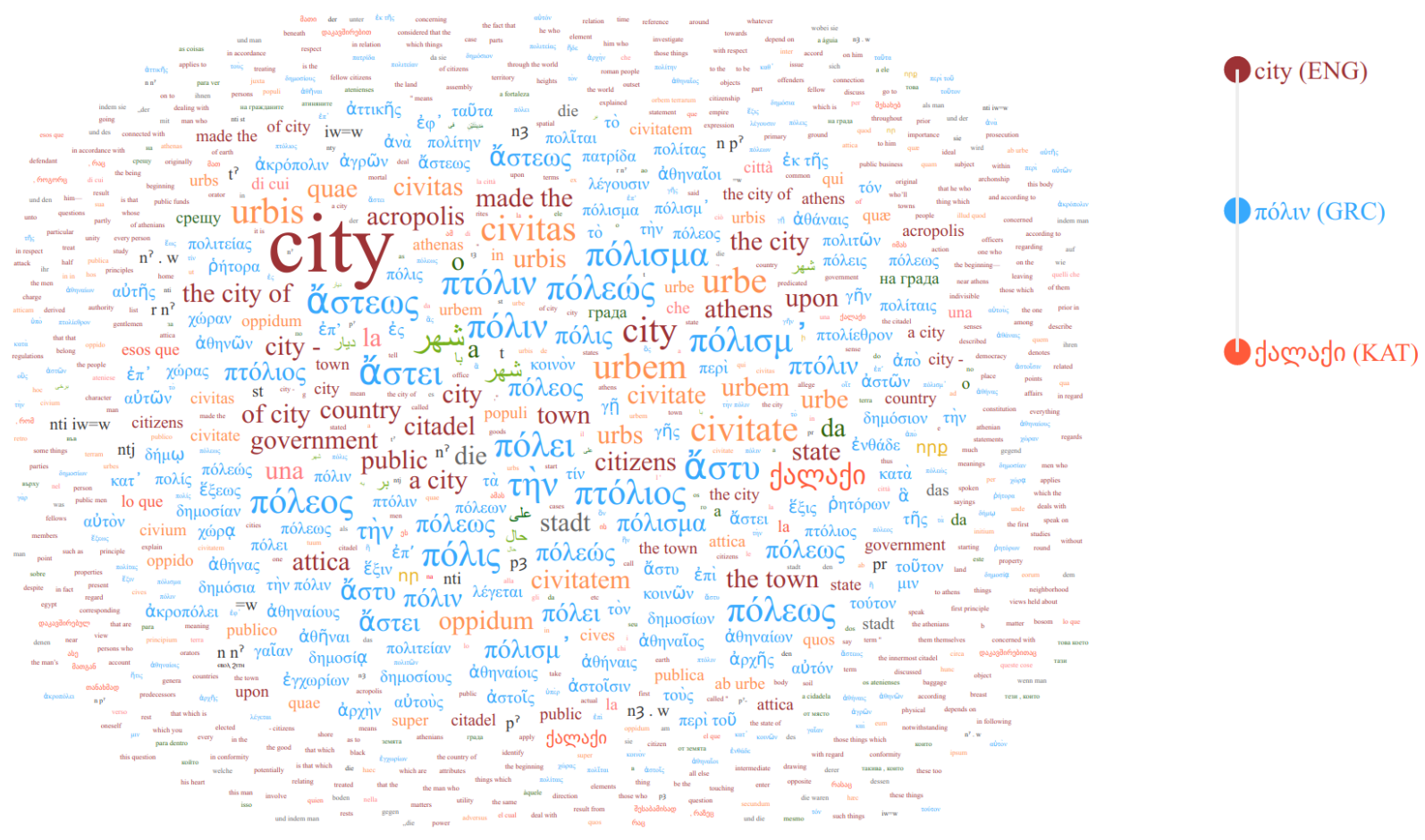

Fig. 11: Translations cloud of the root word city.

possible.

- Automatic alignment: we are currently developing an automatic alignment system and planning to offer an automatic alignment option for texts in specific languages such as Ancient Greek-English and Latin-English or at least supporting the users with alignment suggestions to reduce the time required to align long texts.

- Collaborative alignment: in the current version, users can only align their texts; However, the next version will provide an option for the collaborative alignment of long texts where multiple users can work on the same text.

\section{REFERENCES}

[1] Martin Kay and Martin Röscheisen. Text-translation alignment. Computational Linguistics, 19(1), 1993.

[2] Peter F. Brown, John Cocke, Stephen A. Della-Pietra, Vincent J. DellaPietra, Frederick Jelinek, John D. Lafferty, Robert L. Mercer, and Paul Rossin. A statistical approach to machine translation. Computational Linguistics, 16(2):76-85, 1990.

[3] William A. Gale and Kenneth Ward Church. A program for aligning sentences in bilingual corpora. In Proceedings of the 29th Annual Meeting of the Association of Computational Linguistics (ACL), 1991.

[4] Robert C. Moore. Fast and accurate sentence alignment of bilingual corpora. In Stephen D. Richardson, editor, Machine Translation: From Research to Real Users, 5th Conference of the Association for Machine Translation in the Americas, AMTA 2002 Tiburon, CA, USA, October 6-12, 2002, Proceedings, volume 2499 of Lecture Notes in Computer Science. Springer, 2002.

[5] David Bamman and Gregory Crane. Measuring historical word sense variation. In Proceedings of the 11th Annual International ACM/IEEE Joint Conference on Digital Libraries, JCDL '11, page 1-10, New York, NY, USA, 2011. Association for Computing Machinery.

[6] I. Dan Melamed. Manual annotation of translational equivalence: The blinker project. CoRR, cmp-lg/9805005, 1998.
[7] Stephen Grimes, Xuansong Li, Ann Bies, S. Kulick, Xiaoyi Ma, and Stephanie Strassel. Creating arabic-english parallel word-aligned treebank corpora at ldc. 2010.

[8] H. M. Caseli, V. D. Feltrim, and M. G. V. Nunes. Tagalign: Uma ferramenta de pré-processamento de textos (nilc-tr-02-09). Technical Report 169, 2002.

[9] Bridget Almas and Marie-Claire Beaulieu. Developing a New Integrated Editing Platform for Source Documents in Classics. Literary and Linguistic Computing, 28(4):493-503, 072013.

[10] Bridget Almas and Monica Berti. Perseids collaborative platform for annotating text re-uses of fragmentary authors. 092013.

[11] Juxtacommons. A user guide to juxta commons, 2019. Accessed 10 Sept. 2019.

[12] Stefan Jänicke, Annette Geßner, Greta Franzini, Melissa Terras, Simon Mahony, and Gerik Scheuermann. TRAViz: A Visualization for Variant Graphs. Digital Scholarship in the Humanities, 30(suppl_1):i83-i99, 10 2015.

[13] Tara L. Andrews and Caroline Macé. Beyond the tree of texts: Building an empirical model of scribal variation through graph analysis of texts and stemmata. LLC, 28:504-521, 2013.

[14] Tom Cheesman, Stephan Thiel, Kevin Flanagan, Zhao Geng, Alison Ehrmann, Robert S. Laramee, Jonathan Hope, and David M. Berry. Translation arrays: Exploring cultural heritage texts across languages. In Proceedings of the Digital Humanities 2012, 2012.

[15] S. Jänicke and D. J. Wrisley. Interactive visual alignment of medieval text versions. In 2017 IEEE Conference on Visual Analytics Science and Technology (VAST), pages 127-138, 2017.

[16] M. Pöckelmann, A. Medek, P. Molitor, and J. Ritter. Catview supporting the investigation of text genesis of large manuscripts by an overall interactive visualization tool. In Proceedings of the Digital Humanities 2015, 2015.

[17] Tariq Yousef, Chiara Palladino, and Gregory Crane. Intra-language text alignment using ialigner. In Proceedings, 7th International Conference of Digital Archives and Digital Humanities. National Taiwan University, 122016.

[18] Matthew Petrillo and Jessica Baycroft. Introduction to manual annotation. Fairview research, 2010. 
[19] Alison Babeu. The Perseus Catalog: of FRBR, Finding Aids, Linked Data, and Open Greek and Latin, pages 53-72. 082019.

[20] Tariq Yousef and Monica Berti. The digital fragmenta historicorum graecorum and the ancient greek-latin dynamic lexicon. In Proceedings of the Workshop on Corpus-Based Research in the Humanities (CRH) 2015, Warsaw, Poland, December 2015. Institute of Computer Science, Polish Academy of Science.

[21] Judit Ács. Pivot-based multilingual dictionary building using Wiktionary. In Proceedings of the Ninth International Conference on Language Resources and Evaluation (LREC'14), pages 1938-1942, Reykjavik, Iceland, May 2014. European Language Resources Association (ELRA).

[22] Neo4j Graph Database. Graph modeling guidelines, 2019. Accessed 02 Nov. 2019.

[23] The digital rosetta stone project. https://rosetta-stone.dh.uni-leipzig.de/, 2019. Accessed 02 Nov. 2019.

[24] Maia Shukhoshvili. Methodology of translation alignment of georgian text of plato's "theaetetus". International Journal of Language Linguistics, 2010.

[25] Maryam Foradi, Chiara Palladino, and Farnoosh Shamsian. Confronting complexity of babel in a global and digital age. In DH2019: Digital Humanities Conference, Book of Abstracts., 2019. 